\title{
A FEW FAMILIES OF CAYLEY GRAPHS AND THEIR EFFICIENCY AS COMMUNICATION NETWORKS
}

\author{
HAMID MOKHTAR
}

(Received 30 December 2016; first published online 2 March 2017)

2010 Mathematics subject classification: primary 68M10; secondary 90B18, 90C27.

Keywords and phrases: Cayley graph, circulant graph, cube-connected cycle, interconnection network, routing, shortest path, diameter, Wiener index, edge-forwarding index, vertex-forwarding index, bisection width.

Cayley graphs are highly attractive structures for communication networks because of their many desirable properties, including vertex-transitivity and efficient routing algorithms [4]. The families of circulants and cube-connected graphs are among the most popular Cayley graphs for efficient communication networks [11, 12]. The diameter, forwarding and optical indices, bisection width and Wiener index of a network are among the most important parameters to measure the efficiency of the network [2, 5-7].

Circulant graphs and, in particular, circulant graphs with small degrees are interesting models for communication networks [1]. However, our knowledge of many of their parameters, including the arc-forwarding index, edge-forwarding index, directed and undirected optical indices, are very limited, except for very few special cases. We study the family of circulant graphs of degree 4 and obtain lower and upper bounds for their forwarding and optical indices. We give approximation algorithms for the corresponding problems of the forwarding indices and optical indices with a small constant performance ratio. Our results on the family of circulant graphs of degree 4 are published in [3].

The family of recursive cubes of rings has received a lot of attention for communication networks [10], but many aspects of them have remained unknown. We study this family of graphs by redefining each of them as a Cayley graph on the semidirect product of an elementary abelian group by a cyclic group in order to facilitate the study of them by using algebraic tools. We give an algorithm for computing shortest paths and obtain the exact value of their diameters. We obtain

Thesis submitted to the University of Melbourne in April 2016, degree approved on 10 August 2016, supervisor Sanming Zhou.

(c) 2017 Australian Mathematical Publishing Association Inc. 0004-9727/2017 \$16.00 
sharp bounds on the Wiener index, vertex-forwarding index, edge-forwarding index and bisection width of recursive cubes of rings. The cube-connected cycles and cube of rings are special recursive cubes of rings, and our results apply to these well-known networks. Our results on this topic are published in [9].

We introduce cube-connected circulants as a new family of cube-connected Cayley graphs and study their efficiency for communication networks [8]. We give an algorithm for computing shortest path routing and the exact value of the diameter of a cube-connected circulant. We observe that while recursive cubes of rings are special cube-connected circulants, these two families of cube-connected graphs have significantly different routing behaviours in general. Hence we develop results for 'proportional' graphs which will be useful in obtaining bounds for the edge-forwarding index of cube-connected circulants. We give sharp lower and upper bounds for the Wiener index, vertex-forwarding and edge-forwarding indices of cube-connected circulants. We study the embedding of cube-connected circulants into hypercubes and the embedding of hypercubes into cube-connected circulants. We show that cube-connected circulants outperform a few well-known network topologies in many aspects.

\section{Acknowledgements}

I would like to express my gratitude to Dr Sanming Zhou for his supprotive supervision. I am grateful to the University of Melbourne and its School of Mathematics and Statistics for this academic opportunity and scholarships MIFRS and MIRS.

\section{References}

[1] J. C. Bermond, F. Comellas and D. F. Hsu, 'Distributed loop computer-networks: a survey', J. Parallel Distrib. Comput. 24(1) (1995), 2-10.

[2] J. C. Bermond, L. Gargano, S. Perennes, A. A. Rescigno and U. Vaccaro, 'Efficient collective communication in optical networks', Theoret. Comput. Sci. 223 (2000), 165-189.

[3] H.-S. Gan, H. Mokhtar and S. Zhou, 'Forwarding and optical indices of 4-regular circulant networks', J. Discrete Algorithms 35(4) (2015), 27-39.

[4] M. C. Heydemann, Cayley Graphs and Interconnection Networks (Springer, Dordrecht, 1997).

[5] M. C. Heydemann, J. C. Meyer and D. Sotteau, 'On forwarding indices of networks', Discrete Appl. Math. 23(2) (1989), 103-123.

[6] S. Lakshmivarahan, J. S. Jwo and S. K. Dhall, 'Symmetry in interconnection networks based on Cayley graphs of permutation groups: A survey', Parallel Comput. 19(4) (1993), 361-407.

[7] B. Mans and I. Shparlinski, 'Bisecting and gossiping in circulant graphs', in: LATIN 2004: Theoretical Informatics, Proceedings of the 6th Latin American Symposium, Buenos Aires, Argentina, 2004 (ed. M. Farach-Colton) (Springer, Berlin, 2004), 589-598.

[8] H. Mokhtar, 'Cube-connected circulants: new efficient models for interconnection networks', Preprint.

[9] H. Mokhtar and S. Zhou, 'Recursive cubes of rings as models for interconnection networks', Discrete Appl. Math. 217(3) (2017), 639-662.

[10] Y. Sun, P. Y. S. Cheung and X. Lin, 'Recursive cube of rings: a new topology for interconnection networks', Parallel Distrib. Syst IEEE Trans. 11 (2000), 275-286. 
[11] A. Thomson and S. Zhou, 'Frobenius circulant graphs of valency four', J. Aust. Math. Soc. 85 (2008), 269-282.

[12] J. Xu, Topological Structure and Analysis of Interconnection Networks, Network Theory and Applications, 7 (Springer, New York, 2013).

HAMID MOKHTAR, School of Mathematics and Statistics, The University of Melbourne, Victoria 3010, Australia e-mail: hmokhtar@student.unimelb.edu.au 